\title{
L'archivio don Lorenzo Milani della Fondazione per le scienze religiose di Bologna. Cronistoria di carte e ricerca
}

L'autore descrive i motivi della costituzione dell'Archivio di don Lorenzo Milani a Bologna, presso la Fondazione per le scienze religiose e $i$ cantieri di ricerca attivati, tra cui quello di edizione dell'Opera Omnia degli scritti di Milani, per i Meridiani Mondadori, in uscita nel 2017, per il cinquantennale della morte del priore di Barbiana.

The author describes the reasons for the founding of the Archive Don Lorenzo Milani in Bologna, at the Foundation for Religious Studies, in 1974. In addition, the article outlines the status of the research on Don Milani, including the Opera Omnia of his writings, which will be published in 2017 by Meridiani Mondadori, on the Fiftieth anniversary of his death.

\section{Gli inizi: il 1974. Nasce l'archivio Milani a Bologna}

Don Lorenzo Milani è morto a Firenze il 26 giugno 1967, a 44 anni. Nel giugno del 1974 molte fra le persone che per i più diversi motivi avevano avuto con lui contatti personali o corrispondenza ricevettero questa lettera inviata da sua madre, l'ebrea triestina Alice Weiss:

A sette anni dalla scomparsa di Lorenzo Milani la figura e l'opera di lui costituiscono, e non solo in Italia, una delle testimonianze più rilevanti della vita spirituale del nostro tempo. Infatti i suoi pochi scritti e le lettere già pubblicate meritano $\mathrm{e}$ ottengono una attenzione religiosa, letteraria e civile perché segni di un valore che si precisa e si accresce nel tempo. Per questa ragione, e perché l'eredità della sua parola e del suo esempio non si disperda, bene comune affidato a tutti, si è costituito presso l'Istituto per le Scienze Religiose di Bologna, un Fondo Lorenzo 
Milani, destinato a raccogliere e custodire scritti, lettere, testimonianze che possono direttamente o anche indirettamente illuminare maggiormente la sua figura e la sua opera. Quale primo nucleo di tale Fondo abbiamo voluto donare al Fondo stesso gli originali delle lettere di Don Lorenzo a noi dirette (tanto le edite quanto le inedite) desiderando con ciò dare l'esempio di una volontà che vorremmo condivisa da tutti coloro che posseggono documenti originali o testimonianze relative alla vita e all'opera di Don Lorenzo. Il materiale raccolto in originale o in copia presso l'Istituto potrà essere consultato da studiosi e ricercatori, con vincoli posti eventualmente dai donatori. Ogni ulteriore prezioso contributo potrà essere inviato al Fondo Lorenzo Milani presso la sede dell'Istituto per le Scienze Religiose, via San Vitale 114, Bologna.

La madre dava il buon esempio il dicembre successivo, consegnando nel fondo così costituito 415 manoscritti originali editi e inediti del figlio (per lo più composto dal carteggio privato ricevuto dal figlio), a cui seguirono altre importanti donazioni di suoi allievi e amici (Francuccio, Elena Pirelli Brambilla, Giorgio Pecorini) [Battelli 1980]?

L'iniziativa della famiglia Milani, anche su consiglio di Michele Ranchetti, toccava così un nodo centrale della questione sulla memoria milaniana e allo stesso tempo faceva venire a galla una sensibilità maturata in primis in seno alla famiglia e alla cerchia ristretta di amici, ovvero la necessità di raccogliere in un unico luogo i numerosi documenti del figlio, per sottrarli agli usi personali, per garantire a essi una conservazione nel tempo e, soprattutto, per studiarli in modo serio e critico. La lettera arrivava a pochi anni dalle uscite delle prime due importanti raccolte di lettere di Milani, quelle curate da Michele Gesualdi Lettere di don

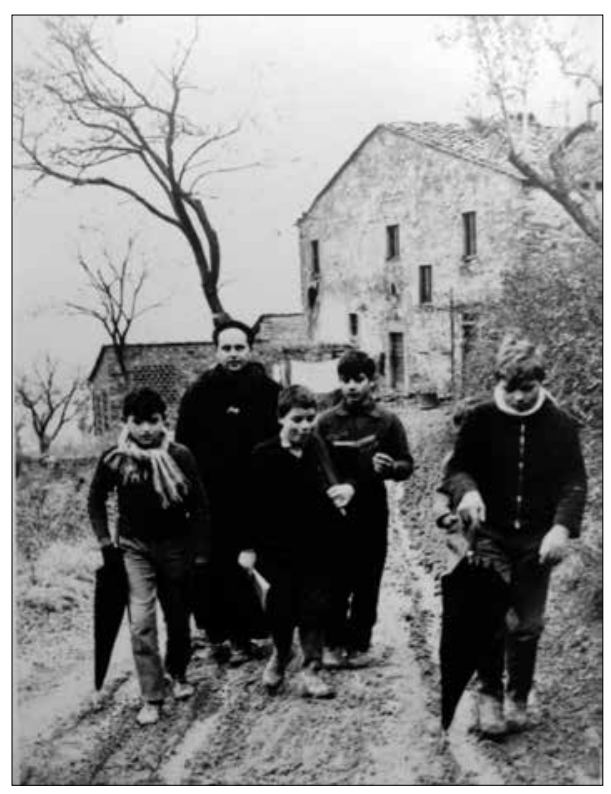

Lorenzo Milani a Barbiana, assieme ai suoi ragazzi (foto di Oliviero Toscani, Archivio Milani, Fscire) Lorenzo Milani al priore di Barbiana (Mondadori 1970), e quelle (selezionate) alla mamma (Mondadori 1973), curate 
dalla stessa Alice'2, grazie all'aiuto intelligente di Donato Barbone, allora editor della casa editrice milanese. Furono entrambi grandi successi editoriali e fecero toccare con mano l'interesse generale verso la figura di don Milani. Tra la primavera e l'estate del 1974 uscivano inoltre uno dietro l'altro altri due volumi, Lorenzo Milani profeta cristiano di Giampiero Bruni, all'interno della collana «I libri di Corea» della Lef, e la sua prima (e ancora di fatto l'unica, per certi versi) biografia, scritta da Neera Fallaci e più volte aggiornata e ristampata (Dalla parte dell'ultimo: vita del prete Lorenzo Milani). Una vera e propria sbornia editoriale, tanto da far scrivere a Dino Pieraccioni su "Il focolare" che «dalla morte di don Lorenzo Milani, priore di Barbiana, avvenuta a Firenze il 26 giugno 1967, son passati appena sette anni. La figura di questo singolare prete fiorentino continua a suscitare interessi sempre più vivi. La bibliografia ormai non si conta»?3.

L'idea di un "archivio Milani” era peraltro già un sentire comune negli ambienti milaniani, proprio sulla scia del florido mercato editoriale che stava interessando la figura di Milani, ormai diventato «oggetto di riconoscimento unanime, fin quasi a non essere più quel che fu in vita, segno di contraddizione» e di riflesso, sulla presa d'atto della «provvisorietà» di questi studi, in balia della caccia agli inediti, della pubblicazione di lettere monche, di censure incomprensibili. Proprio Mario Gozzini su "La Stampa" nel marzo 1973 si era fatto promotore di tale iniziativa, cogliendo l'occasione della presentazione della raccolta mondadoriana di lettere alla mamma:

La provvisorietà degli studi che gli sono stati dedicati [...] fa sentire, da un lato, l'urgenza di una biografia rigorosa, dall'altro la necessità di un'iniziativa pubblica per la costituzione di un archivio delle carte inedite che le sottragga agli umori dei singoli e dei gruppi che le detengono. ${ }^{4}$

Sempre Gozzini continuava rivolgendo alla madre Alice il suo profondo ringraziamento proprio per «aver vinto naturali esitazioni; per aver capito oggi che non appartenevano soltanto a lei queste lettere (pur tanto care), così come aveva capito ieri che nulla le apparteneva del suo grande figliolo se non l'affetto»'.

Qui conviene forse ricordare che quando, alla fine del XVI secolo, si inizia a fare dell'archivio un uso storico e non più solo giuridico, si produce una vera e propria

Battelli [1981, 61] offre «qualche sintetica annotazione sul grado di affidabilità» di queste raccolte: individua infatti, soprattutto nella edizione Lettere di don Lorenzo Milani, priore di Barbiana, «una serie notevolmente estesa di imprecisioni» sia a livello formale, sia a livello di contenuto.

3 D. Pieraccioni, Sette anni dalla morte. Don Milani priore di Barbiana, "Il focolare", 29 settembre 1974.

M. Gozzini, Cara mamma. Altre lettere di don Milani, "La Stampa”, 23 marzo 1973.

Ibidem. 


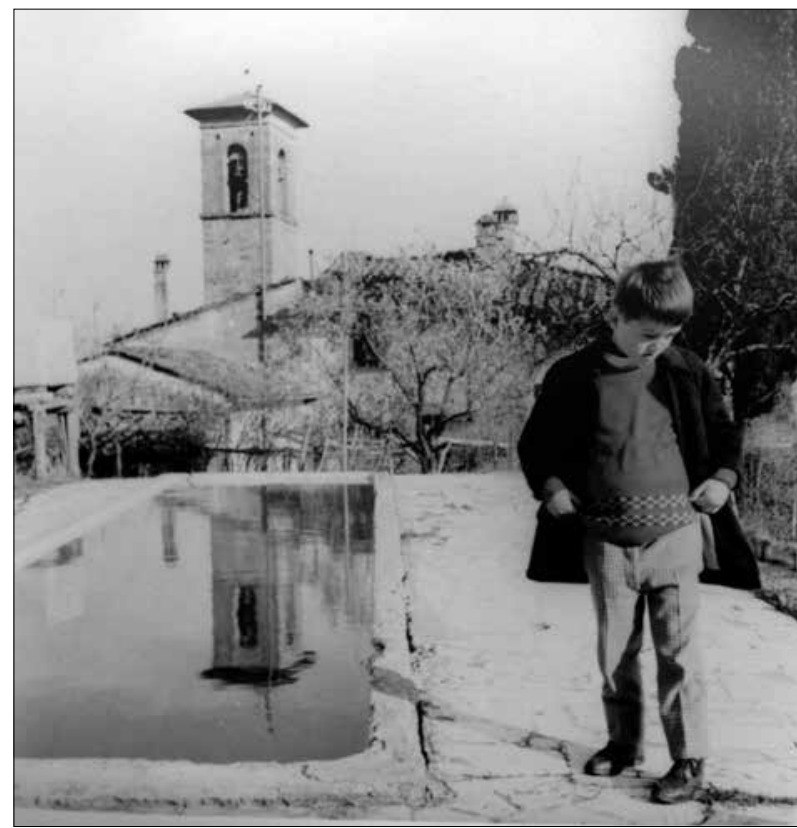

La piscina voluta e costruita da Milani per far perdere la paura dell'acqua ai suoi giovani ragazzi montanari (foto di Oliviero Toscani, Archivio Milani, Fscire). frattura. L'archivio smette di essere memoriaautodocumentazione per diventare memoria-fonte [Zanni Rosiello 2005], «a partire da discorsi che hanno appena cessato di essere nostri», come scriveva Michel Foucault nella sua Archeologia del sapere. Archivio dunque come luogo che conserva, ma anche come luogo che studia ciò che conserva. Non un archivio vetrina, un archivio sotto teca da proteggere, o ancor meno sotto chiave da rendere inaccessibile, bensì da interrogare, da condividere e studiare. Precisamente quello che auspicava la madre di don Lorenzo portando le carte del figlio, ormai reso ancora più celebre sull'onda del fraintendimento Sessantottesco della sua denuncia della scuola borghese e del suo "non bocciare", nel centro di ricerca fondato da Giuseppe Dossetti nel 1953.

Che cosa fosse l'allora Istituto per le scienze religiose di Bologna (oggi Fondazione), soprattutto per coloro che non erano addentro agli studi storici, lo spiegava Paolo Pombeni, allora giovane ricercatore di tale istituzione e responsabile dell'archivio Milani, rispondendo ad un articolo di Maurizio Di Giacomo, il quale, in una lunga panoramica sulla questione degli inediti milaniani e sulle problematiche riscontrate ad essi connessi (tagli, etc.), dava notizia della costituzione del fondo bolognese, avanzando però alcuni interrogativi, tra i quali l'indipendenza di questo istituto di ricerca dalle eventuali pressioni di origine ecclesiastica ${ }^{6}$.

Oltre a ridimensionare la dietrologia romanzesca nel descrivere la vicenda degli 
inediti - «a chi ha dimestichezza con la raccolta di fonti circa la storia contemporanea (religiosa e non), parrà incredibile fortuna che a così pochi anni dalla morte di don Milani ci sia già un numero così consistente di carte conservate e sottratte alle vicende personali di questo o di quello; e parrà ancora più incredibile che, pur con qualche modesto taglio, sia disponibile già una edizione di molto di questo materiale» ${ }^{\prime}$-, rispondeva all'insinuazione delle influenze esterne descrivendo la natura che ancora oggi muove la ricerca in questo istituto e che ne ha fatto un prestigioso centro di eccellenza internazionale della ricerca storico-religiosa.

\section{Dopo le carte, il cantiere di ricerca su don Milani della Fon- dazione: ieri, oggi e domani}

I primi lavori della Fondazione su Milani furono chiaramente volti ad ordinare e inventariare il materiale arrivato (lettere private, articoli, bozze delle opere, rassegna stampa), cercando di ricreare i tradizionali strumenti di lavoro dello storico: nel 1980, sul secondo numero della rivista "Cristianesimo nella storia", la rivista internazionale della Fondazione fondata all'inizio del 1980 che si occupa di ricerche storiche, esegetiche e teologiche, Giuseppe Battelli pubblicò l'inventario e il regesto dell'epistolario di don Milani, dopo aver descritto il fondo nella prima relazione pubblica presentata ad aprile di quello stesso anno - Fonti per lo studio della figura e dell'opera di Don Lorenzo Milani - all'interno del celebre convegno su Milani tenutosi a Firenze. Nel settembre del 1987, Alberto Melloni e Battelli misero a frutto la fertile intersezione creatasi dal lavoro informatico applicato alle scienze umane e in particolare alle discipline storico-religiose. Dopo la lungimirante iniziativa del gesuita $\mathrm{p}$. Roberto Busa, che più di quarant'anni prima era riuscito a convincere l'IBM statunitense a finanziare l'applicazione dei calcolatori al lessico di Tommaso d'Aquino, dando vita all'utilissimo Index Thomisticus ${ }^{8}$, anche la Fondazione si impegnò dunque in questo filone con le prime ricerche sui corpora, grazie al connubio tra tecnologie informatiche, fonti cristiane e autori contemporanei. Si arrivò così nel 1987 a quattro grossi volumi di concordanze, per studio, delle lettere milaniane, ovvero il repertorio alfabetico delle parole di un'opera (in questo caso dell'epistolario), con l'indicazione e la citazione dei luoghi in cui esse ricorrono. Qualche anno prima, nell'ottobre 1985, erano giunte a conclusione quelle degli scritti di Roncalli/Giovanni XXIII [Melloni 1986],

P. Pombeni, Intervento dei lettori, "Idoc Internazionale", maggio 1976.

Per una panoramica, con riferimenti anche all'attività della Fondazione, cfr. Melloni 1997, 129-143. 


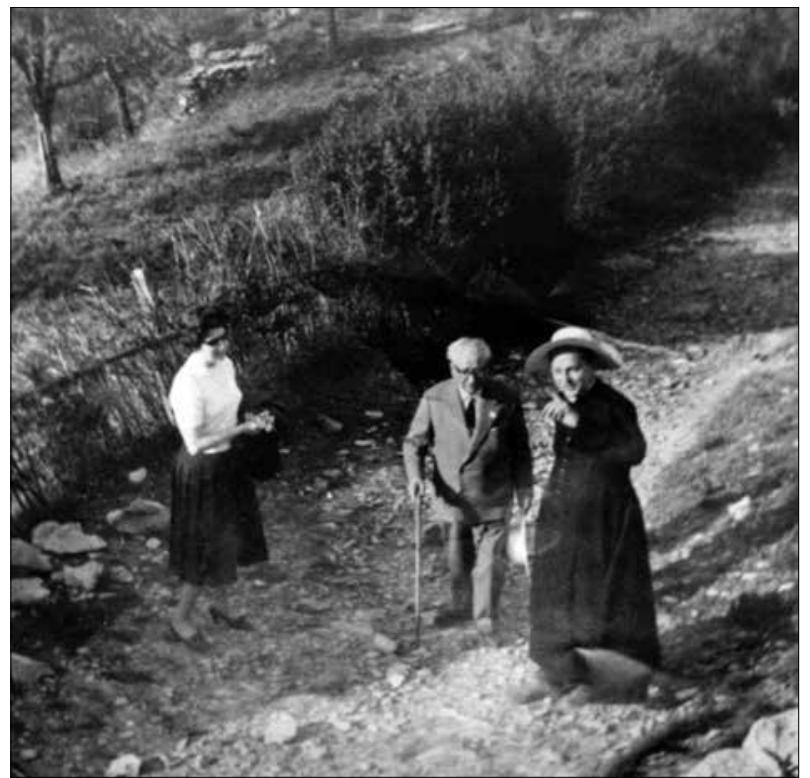

Amici in visita a Barbiana (Archivio Milani, Fscire).

che avevano rappresentato un buon banco di prova per la stessa Fondazione, anch'esse propedeutiche a tutti i lavori di carattere biografico o tematico (13.000 pagine di testi editi più qualche inedito per 22.287 pagine di concordanza).

Quando uscì nel 1990 l'edizione critica delle lettere alla mamma (questa volta integrali) curata da Battelli e uscita per Marietti nel 1990, all'interno della collana della Fondazione (Testi e ricerche

di scienze religiose, n. 5), divennero visibili i primi risultati di un lungo lavoro preparatorio sull'archivio, a cui seguì nel 1994 il lavoro di Massimo Toschi su Esperienze Pastorali (Don Lorenzo Milani e la sua chiesa. Documenti e studi). Il primo rendeva così pubblico tutto il carteggio tra Lorenzo e la madre, già pubblicato parzialmente, in modo lodevole, come detto, nel 1973. Battelli questa volta offriva invece ai lettori 432 lettere, di cui 256 inedite (tutte conservate presso l'Archivio della Fondazione, in versione originale, manoscritta e/o dattiloscritta) ma soprattutto ne correggeva gli errori di trascrizione, ripristinava i tagli nel testo (edito con omissis), gli aggiustamenti linguistici e sintattici che di volta in volta erano stati apportati, le omissioni di nomi e offriva un apparato di note con tutta una serie di informazioni a corollario che permettevano così una maggiore comprensione e contestualizzazione di quell'epistolario e della sua scrittura, che Fortini [1981, 183] definì come «oratoria sacra»". Il secondo, invece, ricostruiva i rapporti tra il priore di Barbiana e la chiesa fiorentina, possibile grazie ai nuovi documenti venuti alla luce e conservati nell'archivio della diocesi, a cui si aggiungevano copie della corrispondenza tra curia, Santa Sede e Sant'Offizio (tutti documenti ora in copia nell'archivio Milani bolognese).

Dopo la lunga stagione di studio del Concilio Vaticano II e di papa Giovanni 
XXIII, che ha impegnato per più di un decennio le forze della Fondazione a livello internazionale nel tentativo di storicizzare quell'evento e arrivando alla produzione di cinque volumi (tradotti in sette lingue) ormai celebri - La storia del concilio Vaticano II diretta da Giuseppe Alberigo e curata da Melloni - il cantiere di ricerca su don Milani è continuato negli anni Duemila, con nuove forze e progetti, sempre con l'idea di scrostare dalla figura di don Milani pregiudizi e apologie che rischiavano, ieri come oggi, di azzerare e di annacquare la forza e la tipicità dell'opera del prete fiorentino. Il lavoro di risistemazione e di inventariazione delle carte ha portato dunque nel quarantennale della morte di don Lorenzo alla realizzazione di un documentario biografico trasmesso su Raiuno, Lorenzino don Milani (all'interno degli Speciali TV7) curato da Melloni, Fabio Nardelli e Federico Ruozzi, che ha segnato di fatto la nuova fase di lavoro, anche sul piano della divulgazione. In questi anni si sono aperte anche nuove collaborazioni con i vari gruppi milaniani, in particolare con il Centro Formazione e Ricerca Don Lorenzo Milani e Scuola di Barbiana di Vicchio e con la Biblioteca del comune che hanno portato ad esempio alla pubblicazione nel 2010 del volume curato da Liana Fiorani Don Lorenzo Milani. Il destino di carta. Rassegna stampa 1949-2005, per i tipi del Mulino, all'interno della collana della Fondazione dedicata all'edizione di fonti (Fonti e strumenti di ricerca). Il volume di 900 pagine raccoglie l'inventario di tutti gli articoli di e su don Milani in 56 anni e, novità non trascurabile, ne offre integralmente $\mathrm{i}$ testi in versione digitale e ricercabile.

Esattamente l'anno prima, nel 2009, appoggiati e sollecitati dagli stessi Giorgio Pecorini, da Miguel Martì e da José Louis Corzo affinché la Fondazione riprendesse le redini di quell'impegno e di quel cantiere milaniano e d'accordo con il segretario della Fondazione, si decise il testo di una lettera da far recapitare, ancora una volta, «agli amici di don Milani», esattamente come fece la madre nel 1974. Il nuovo appello era in linea

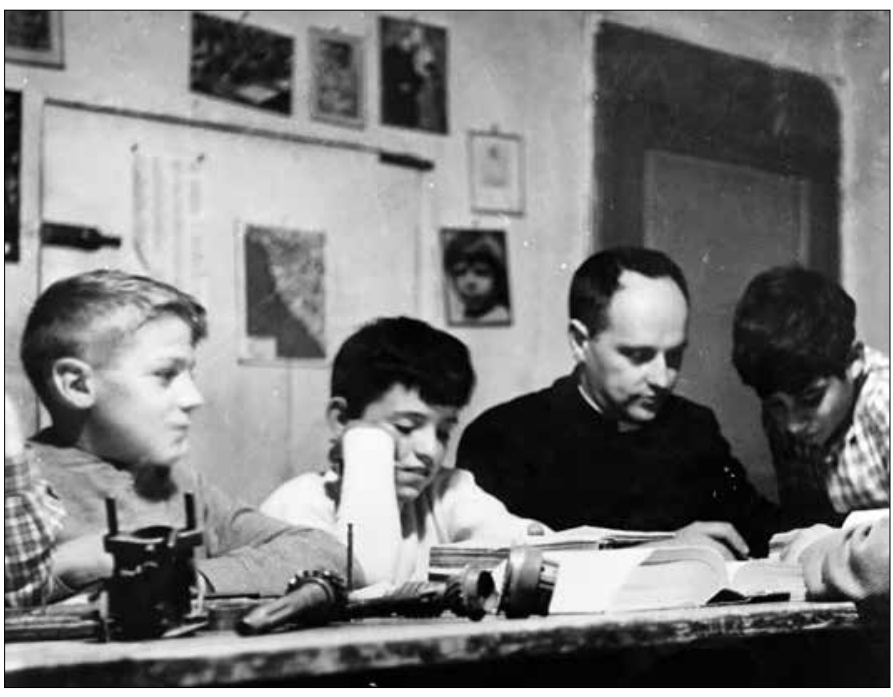

Lezione alla scuola di Barbiana (Archivio Milani, fondo Elena Milani, Fscire). 
con le motivazioni profonde che avevano mosso la madre e i familiari di don Milani allora, e che muovevano ora quel gruppo di studiosi milaniani, ovvero «evitare che sulle prime generazioni di studiosi di don Milani gravi l'onta di aver consegnato al futuro frammenti di lavoro».

A conclusione di quella riunione (7 luglio 2009) che cercò di riunire attorno a un tavolo tutti coloro che, ha diverso motivo, individualmente o come gruppi o istituzioni, giocavano un ruolo nel coltivare la memoria di don Lorenzo, venne fondato un consorzio aperto ${ }^{10}$. Scopo di tale consorzio era «di favorire la collaborazione di studio e lavoro, per evitare di lasciare a maggese il patrimonio di scrittura e di fede di don Lorenzo». I cardini su cui si muoveva tale collaborazione erano, in primis, continuare la raccolta delle carte di don Lorenzo sparse in vari archivi, pubblici e privati, la creazione di un archivio virtuale degli scritti e delle immagini di e su don Milani, da codividere nel rispetto dei vincoli che ciascuno avrebbe fissato e lavorare all'edizione critica dell'opera omnia milaniana, come già si richiedeva da decenni.

\section{Verso un archivio unico digitale}

A distanza di poco meno di quarant'anni, le tecnologie, ancora una volta, rendevano attualizzabile ciò che prima era anche solo difficile pensare, esaudendo così il desiderio della madre e di tanti suoi amici di riunire in un unico archivio le carte del figlio, proprio per evitare dispersioni che il tempo avrebbe certamente incrementato. Se fino ad allora quei desiderata avevano incontrato notevoli difficoltà, anche pratiche, l'informatizzazione le rendeva ora possibili in ambiente digitale. Certamente, in questa trasposizione digitale occorre tenere ben presente la salvaguardia «del contesto archivistico nel quale [quelle fonti documentarie] sono incardinate» [Vitali 2004, 102], nel rispetto del documento, del supporto e dell'apparato filologico.

Il grande vantaggio della riproduzione digitale dei documenti e dell'archivio è inoltre quello di permettere di superare le naturali ritrosie dovute a gelosie, a difficoltà a «consegnare per sempre» un ricordo che di fatto è personale. La scansione delle carte rende infatti superabili tutti questi ostacoli, perché la copia digitale ad alta qualità lascia di fatto l'originale al naturale possessore, che comunque ne può 
decidere l'accesso e le eventuali restrizioni sulla consultazione. La copia digitale garantisce così la conservazione nel tempo del documento e allo stesso tempo la possibilità di interrogare queste fonti in modi nuovi (inventari elettronici, facilità di comparazioni e di ricerche interne, associazione di metadati a corollario) e, appunto, la condivisione di tali documenti in modalità intranet, in remoto. Ci si sta dunque orientando verso una completa migrazione digitale dell'archivio, un vero e proprio archivio Milani strutturato "a rete", su più sedi a livello fisico, ma unico a livello digitale: i vari archivi locali e/o privati continuano ad avere i loro fondi archivistici, i quali tuttavia sono condivisi all'interno della piattaforma digitale, con il risultato di avere un unico grande archivio che un domani (a seconda dei vincoli posti da ciascuno dei donatori) potrà essere consultato direttamente dal web. D'altra parte, è evidente che l'organizzazione del materiale archivistico stia

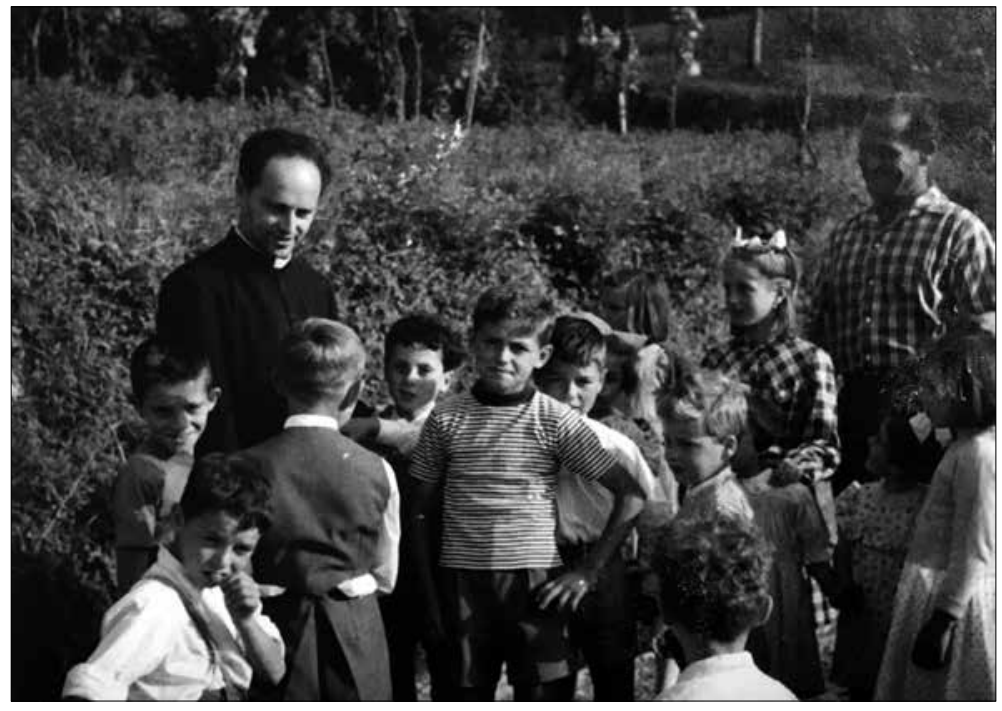

Lezione alla scuola di Barbiana (Archivio Milani, fondo Elena Milani, Fscire).

profondamente cambiando, ed è dunque naturale che ci si allinei agli standard che garantiscano una maggiore accessibilità e fruibilità.

Su queste basi e in continuità con l'investitura della madre Alice Weiss, la Fondazione ha avviato una intensa raccolta di nuovi documenti, tramite incontri e appelli pubblici a tutti coloro che fossero ancora in possesso di carte a fornirne una copia (nuovi carteggi, dattiloscritti e manoscritti parziali di testi milaniani, opere, schizzi, poesie, temi). Così è avvenuto ad esempio l'11 dicembre 2010, presso la biblioteca comunale di Vicchio, in collaborazione con l'Istituzione culturale don Lorenzo Milani e Scuola di Barbiana, il Centro di documentazione don Milani e il centro di formazione e ricerca. L'archivio Milani di Bologna si è così arricchito di nuove e ulteriori donazioni di materiali, a partire da quelle fatte 
dagli ex allievi e amici Enrico Zagli, Nevio Santini, Bruno Becchi, la Biblioteca di Vicchio, Francesca Ichino, Erseo Polacco marito di Elena Milani, sorella di Lorenzo e morta nel 2010 (documenti scritti e l'intero patrimonio fotografico della famiglia Milani composto da più di 3.000 fotografie, integralmente digitalizzato), il fondo Cartoni-Di Giacomo (in copia grazie all'Istituto Storia del cristianesimo di Napoli - Pontificia Facoltà Teologica dell'Italia Meridionale) e da ultimo Giorgio Pecorini, amico di Milani, allora giornalista dell'"Europeo" e ora scrupoloso custode della memoria di don Lorenzo, che ha consegnato alla Fondazione l'intero suo archivio su don Lorenzo, completando così quella prima consegna di documenti in copia fatta negli anni Settanta. Si approfitta tra l'altro di questa occasione per ringraziare pubblicamente tutti coloro che in questi anni hanno aderito a tale progetto e rinnovando l'invito a chi ancora non lo avesse fatto a contattare la Fondazione per eventuali nuove consegne"

Per un aggiornamento di come negli ultimi anni si è arricchito il fondo, si può consultare oggi uno strumento essenziale, che molti chiedevano da tempo, ovvero la cronotassi dettagliata degli scritti di don Lorenzo Milani [Corzo e Ruozzi 2012], apparsa sulla rivista “Cristianesimo nella storia” nell'aprile $2012^{12}$.

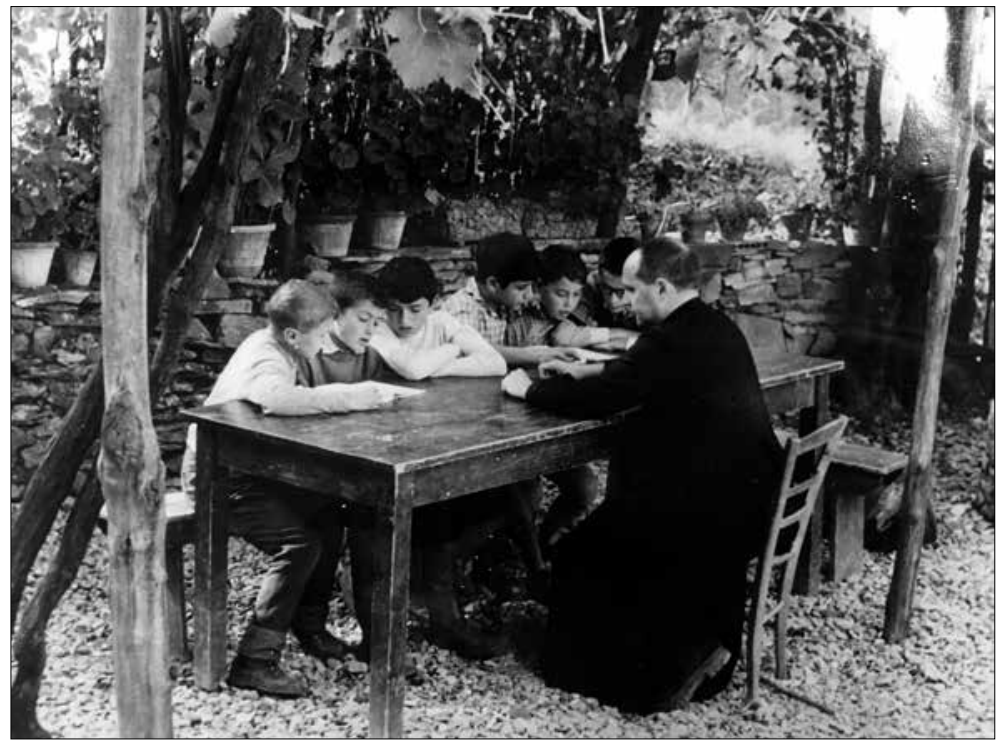

Lezione alla scuola di Barbiana (Archivio Milani, fondo Elena Milani, Fscire).

Nel caso, spedire all'attenzione di Federico Ruozzi, Fondazione per le scienze religiose Giovanni XXIII, via San Vitale, 114,40125 Bologna.

12 Si segnala inoltre Corzo 2014, con un'appendice documentale inedita curata dallo stesso Corzo e da Ruozzi. Quest'ultimo dal 2015 cura anche un bollettino bibliografico periodico, che dà conto in chiave critica delle numerose pubblicazioni che riguardano il priore di Barbiana. 


\section{Un archivio per la memoria, un archivio per la storia}

Se l'intento primario della Fondazione per le scienze religiose nella costituzione di un archivio unico digitale di Lorenzo Milani è quello di preservare la documentazione e dunque la memoria nel tempo, sottraendola a usi e abusi privati, è anche vero che alle fasi della raccolta, della conservazione ed edizione di fonti segue necessariamente quella dello studio. La Fondazione è innanzitutto un istituto di ricerca. La raccolta dei documenti non è dovuta a un puro feticismo delle carte, bensì rappresenta l'unico modo per dare la possibilità ad una comunità di studiosi di ricostruire - nel caso di don Milani - una vita e le sue opere, tenendo sempre ben presente la difficoltà nel fissare sulla pagina la complessità e l'eterogeneità di una esperienza umana. E il lavoro di studio a monte è garanzia della serietà con cui viene svolto.

Per questo la Fondazione da alcuni anni ha avvita il progetto di edizione critica dell'intero corpus milaniano (che coinvolge, oltre ad alcuni studiosi della Fondazione, giovani ricercatori e la Pontificia Facoltà Teologia dell'Italia Meridionale, sezione San Luigi). Ciò ha permesso inoltre di passare al setaccio nuovi e vecchi archivi, trovando ulteriori carteggi, più o meno consistenti, nuove carte (versioni provvisorie, bozze degli articoli, etc.). L'Opera Omnia degli scritti di don Lorenzo Milani ha avuto il riconoscimento dal Mibact che ha inserito l'iniziativa all'interno delle Edizioni Nazionali. Prevista in uscita nel 2017, all'interno dei Meridiani Mondadori, è anche il tentativo di restituire il personaggio Milani e i suoi testi (per la prima volta raccolti insieme) al mondo religioso, culturale e letterario italiano. Don Milani non è infatti stato solo un prete, un educatore, un maestro, ma anche - come si evince dai suoi numerosi scritti, pubblici e privati - uno scrittore.

Ormai a cinquant'anni dalla morte di don Lorenzo, appaiono del tutto anacronistici certi atteggiamenti che, dietro al paravento dell'antintellettualismo, pretendono di "gestire" in senso unico la memoria di don Milani. Fu lo stesso Pecorini che già nel 1968, in occasione del dibattito che nacque tra gli ex alunni in merito alla pubblicazione della prima raccolta di lettere che sarebbe stata pubblicata presso Mondadori due anni dopo (quella dove nella introduzione firmata «I ragazzi di Barbiana» si precisava che «le 127 lettere qui pubblicate sono scelte tra le oltre mille finora raccolte»), scrisse a Michele Gesualdi e a «tutti gli interessati» che: «se ci sembra giusto invece aprire il nostro tesoro, la nostra parte con quella altrui, e spartirlo con tutti gli altri facciamolo ma senza riserve, senza furbizie, senza presunzioni [...] Troppo comodo cavarsela togliendo i documenti contradditori. Cerchiamo piuttosto di offrire ai lettori, a colpi di nota, tutti gli elementi 
per spiegare e per capire. E se non li trovassimo non importa, avrà sempre più ragione lui a contraddirli che noi a censurarlo».

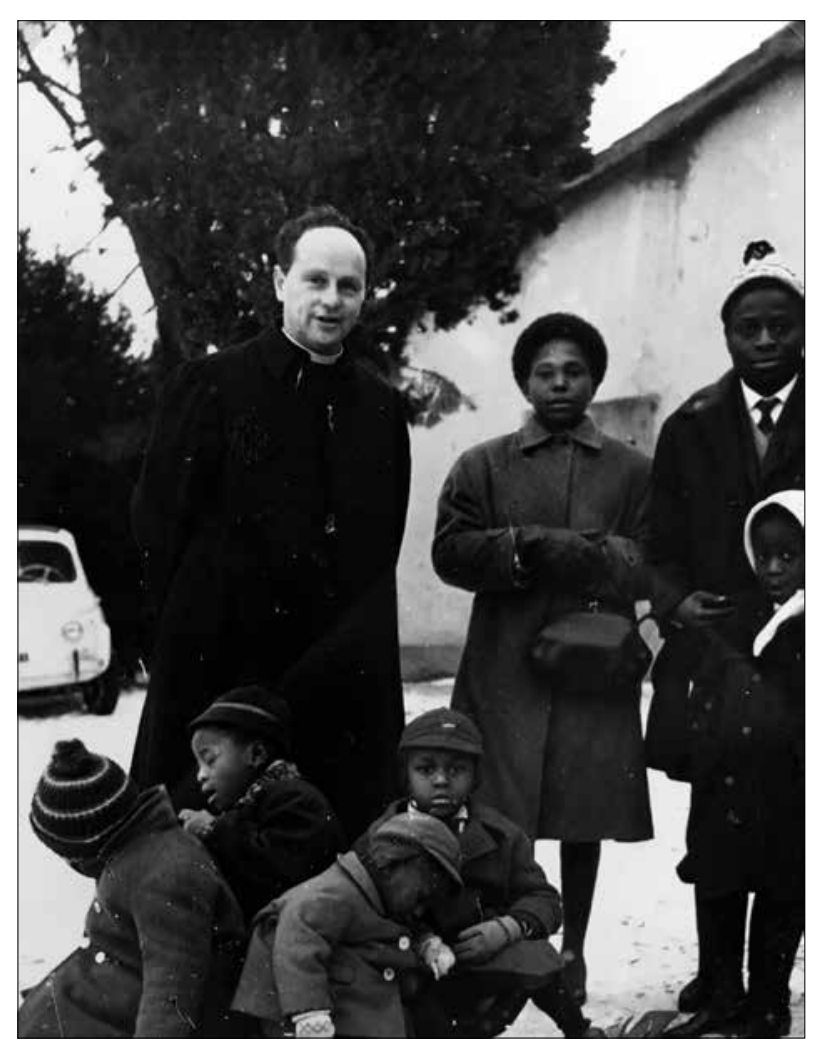

Ospiti a Barbiana (Archivio Milani, Fscire).

L'unico modo per far capire don Milani alle generazioni future è coglierlo nella sua interezza, senza lasciarsi intimorire dalle sue asperità e durezze e, ossimoricamente, dalla sua coerenza. Per questo non si può isolare il don Lorenzo priore di Barbiana dal don Lorenzo cappellano a San Donato; non si può isolare il don Lorenzo maestro dal Lorenzo "scapigliato" a Milano e all'Accademia di Brera da quello in seminario, come non si può ignorare le origini della sua famiglia né tantomeno la cultura che respirava tra i muri di casa. $\mathrm{E}$ questo don Milani ce lo restituisce certo la testimonianza di chi lo ha conosciuto, ma ancora di più i suoi scritti nella loro forma originale e lo studio. Non si può negare che le pagine biografiche della Fallaci, i volumi su Esperienze Pastorali di Toschi e Tanzarella, le analisi di Corzo sulla spiritualità e pedagogia milaniana, le riflessioni di Ranchetti, hanno apportato un contribuito decisivo a far "comprendere" la figura di don Lorenzo, che non si può liquidare sbrigativamente come interessi dell'accademia. Lo storico vive delle carte, ma non ne è schiavo. È schiavo della verità, che cerca di perseguire con il suo lavoro e con il suo studio. È vero che, come qualcuno ha già detto, è l'«ospite non invitato» [Yerushalmi 1983, 105] e la storia molto spesso tende a «sconvolgere» la memoria. Tuttavia, come scriveva Henri-Irénée Marrou [1962, 248], solo al moralista la «storia apparirà come frutto di una semplice curiosità». 


\section{Bibliografia}

Battelli G. 1980, L'epistolario di d. Lorenzo Milani. Inventario e regesto, "Cristianesimo nella storia", 1.

Battelli G. 1981, Fonti per lo studio della figura e dell'opera di don Lorenzo Milani, in Don Lorenzo Milani. Atti del Convegno di Studi (Firenze, 18-19-20 aprile 1980), Firenze: Tip. Nazionale.

Corzo J.L. e Ruozzi R. 2012, Cronotassi degli scritti di don Milani (1928-1967), “Cristianesimo nella storia", 1.

Corzo J.L. 2014, Lorenzo Milani. Una catequesis cronológica sobre la Vida de Jesús, "Cristianesimo nella storia", 3.

Fortini F. 1981, La scrittura di Lorenzo Milani, in Don Lorenzo Milani. Atti del Convegno di Studi (Firenze, 18-19-20 aprile 1980), Firenze: Tip. Nazionale.

Marrou H.-I. 1962, La conoscenza storica, Bologna: il Mulino.

Melloni A. 1986, Concordanza degli scritti di A.G. Roncalli/Giovanni XXIII, "Cristianesimo nella storia", 2.

Melloni A. 1997, Archivi storico-religiosi e strumenti di lavoro. Problemi ed esperienze di coniugazione di tecnologia e ricerca in Italia, in Morelli M. e Ricciardi M. (a cura di), Le Carte della memoria. Archivi e nuove tecnologie, Roma-Bari: Laterza.

Milani L. 1990, Alla mamma. Lettere 1943-1967, edizione critica a cura di Battelli G., Genova: Marietti.

Pecorini G. 1996, Don Milani! Chi era costui?, Milano: Baldini\&Castoldi.

Pecorini G. 2001, I Care ancora, Bologna: EMI.

Toschi M. 1994, Don Lorenzo Milani e la sua chiesa. Documenti e studi, Firenze: Edizioni Polistampa.

Vitali S. 2004, Passato digitale. Le fonti dello storico nell'era del computer, Milano: Bruno Mondadori.

Yerushalmi Y.H. 1983, Zakhor. Storia ebraica e memoria ebraica, Parma: Pratiche.

Zanni Rosiello I. 2005, Gli archivi tra passato e presente, Bologna: il Mulino. 